\title{
First reported case of intraventricular tigecycline for meningitis from extremely drug-resistant Acinetobacter baumannii
}

\author{
*Liverana Lauretti, MD, ${ }^{1}$ Quintino Giorgio D’Alessandris, MD, PhD, ${ }^{1}$ Massimo Fantoni, MD, ${ }^{2}$ \\ Tiziana D'Inzeo, MD, ${ }^{3}$ Eduardo Fernandez, MD, ${ }^{1}$ Roberto Pallini, MD, PhD, ${ }^{1}$ and \\ Giancarlo Scoppettuolo, MD²
}

Institutes of ${ }^{1}$ Neurosurgery, ${ }^{2}$ nfectious Diseases, and ${ }^{3}$ Microbiology, Università Cattolica del Sacro Cuore, Rome, Italy

The authors report on the first case of the intraventricular administration of tigecycline described in the Western literature. A 22-year-old man developed cerebrospinal fluid infection from an extremely drug-resistant strain of Acinetobacter baumannii as a complication of endoscopic transsphenoidal surgery for the removal of a giant pituitary adenoma. The patient was safely and successfully treated with the prolonged intraventricular administration of tigecycline. Here, the authors provide the schedule details that can be invaluable in treating meningitis from extremely drug-resistant bacterial strains. Intraventricular tigecycline can be a valuable tool against multidrug-resistant central nervous system infections. https://thejns.org/doi/abs/10.3171/2016.6.JNS16352

KEY WORDS transsphenoidal surgery; meningitis; Acinetobacter; tigecycline; intraventricular therapy; infection

$\mathrm{C}$ ENTRAL nervous system (CNS) infections from Acinetobacter baumannii represent $3.6 \%-11.2 \%$ of cases of nosocomial meningitis; ${ }^{6}$ those caused by multidrug-resistant (MDR) or extremely drug-resistant (XDR) strains carry a very dismal prognosis, with mortality rates up to $71 \%{ }^{5,8}$ Intraventricular (IVT) and intravenous (IV) colistin is currently the treatment of choice,,$^{1,2,4,5,8}$ but the incidence of neurotoxicity is not negligible (up to 21.7\%). ${ }^{6}$ Moreover, the onset of colistin-resistant Acinetobacter is a well-recognized and hazardous event. ${ }^{7}$ Reportedly, tigecycline has excellent activity against Acinetobacter,${ }^{5,12}$ shows synergy with many antimicrobial agents, ${ }^{3}$ and has a favorable toxicity profile,$^{12}$ particularly as regards nervous system adverse events. Unfortunately, tigecycline CNS penetration is insufficient; $, 6,8,11,14$ therefore, its IV use is not recommended for Acinetobacter meningitis. ${ }^{6}$ These data would prompt IVT tigecycline administration for treating meningitis from XDR bacteria; in the literature, however, there are no reports on such a use, except for a single study in which no treatment details are available. ${ }^{15}$
Here, we describe the first case of the successful IVT use of tigecycline to treat meningitis from colistin-resistant Acinetobacter and also provide a detailed therapeutic schedule.

\section{Case Report}

Surgery for Pituitary Adenoma

A 22-year-old man was admitted to our institution in May 2014 with a 1-month history of headache and progressive visual loss in his left eye. Ophthalmological examination revealed visual acuity $1 / 20$ in the left eye, 20/20 in the right eye, and bitemporal hemianopia. Neuroimaging showed a pituitary adenoma (40-mm maximum diameter) with optic chiasm compression, erosion of the sellar floor, and parasellar extension to the right cavernous sinus (Fig. 1A). Analysis of serum pituitary hormones showed mild hypopituitarism. The patient underwent surgery via a transsphenoidal endoscopic endonasal approach with complete tumor removal. Perioperative IV antibiotic prophylaxis with cefazolin $(2 \mathrm{~g}$ ) and metronidazole (500 mg)

ABBREVIATIONS CNS = central nervous system; CSF = cerebrospinal fluid; IV = intravenous; IVT = intraventricular; $M D R=$ multidrug resistant; $X D R=$ extremely drug resistant.

SUBMITTED February 12, 2016. ACCEPTED June 6, 2016.

INCLUDE WHEN CITING Published online August 19, 2016; DOI: 10.3171/2016.6.JNS16352.

* Drs. Lauretti and D'Alessandris contributed equally to this work. 

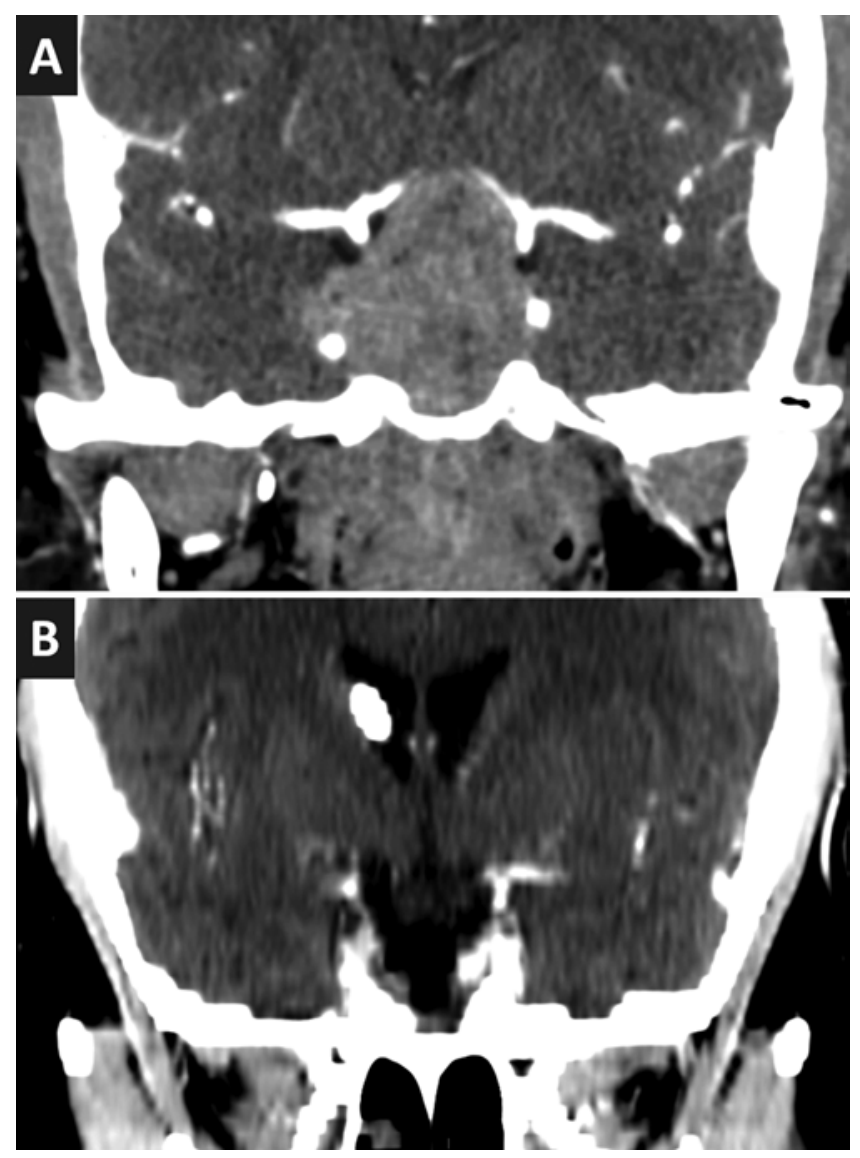

FIG. 1. A: Contrast-enhanced CT scan, coronal reconstruction, showing a giant pituitary adenoma (maximum diameter $40 \mathrm{~mm}$ ) with suprasellar and parasellar extension to the right cavernous sinus. B: Contrastenhanced CT scan, coronal reconstruction, showing removal of the adenoma as well as the implanted right frontal ventricular catheter for IVT therapy.

was administered. Intraoperatively, no cerebrospinal fluid (CSF) leak was detected; the sella was packed using Gelfoam because of prolapse of the diaphragma sellae. Histological examination revealed a nonfunctioning adenoma. The patient's postoperative course was uneventful with remarkable improvement in visual function (10/20 in the left eye). He was discharged by 4 days after surgery, and steroid replacement therapy was prescribed.

\section{Cerebrospinal Fluid Leak and Meningitis}

Two weeks after discharge, the patient came back to the emergency room because of headache and CSF rhinorrhea. Computed tomography scanning revealed pneumocephalus. To counteract the CSF leakage, 3 repeated transsphenoidal procedures were necessary, in which fibrin glue, dural substitutes, autologous fat tissue graft, and temporary lumbar drainage were used. In the meantime, meningitis had clearly developed. Cerebrospinal fluid cultures revealed a pandrug-resistant strain of Acinetobacter baumannii, according to European Committee on Antimicrobial Susceptibility Testing (EUCAST) criteria (http:// www.eucast.org/fileadmin/src/media/PDFs/EUCAST_ files/Breakpoint_tables/Breakpoint_table_v_4.0.pdf), and a minimum inhibitory concentration to tigecycline of 2 $\mu \mathrm{g} / \mathrm{ml}$ (Table 1). Blood cultures were negative. Intravenous tigecycline (100 mg twice daily) was started, as were IV meropenem ( $2 \mathrm{~g}$ thrice daily) and vancomycin ( $1 \mathrm{~g}$ twice daily).

\section{Intraventricular Therapy}

Though the CSF fistula was clinically and radiologically sealed off, CSF cultures remained positive for Acinetobacter. Therefore, off-label IVT tigecycline administration was proposed as the only available therapeutic option given the poor blood-brain barrier permeability to tigecycline. The Institutional Ethics Committee of the Università Cattolica del Sacro Cuore approved this treatment, and the patient signed the informed consent form. Then, an external catheter was implanted in the frontal horn of the right lateral ventricle (Fig. 1B), and IVT tigecycline was started at a dose of $2 \mathrm{mg} / \mathrm{day}$. Tigecycline was diluted in saline up to a total volume of $1 \mathrm{ml}$ and slowly injected into the CSF. After every injection, the CSF drain was temporarily closed for 2 hours to prevent untimely washout of the drug. Intraventricular treatment was well tolerated by the patient. Intravenously, the tigecycline was continued, whereas the meropenem and vancomycin were discontinued. Unfortunately, after fugacious clinical and laboratory improvement, 10 days from the start of the IVT tigecycline, the bacterial load increased again and fever and rigor recurred. Thus, the IVT tigecycline dose was escalated to $2 \mathrm{mg}$ twice daily ( $4 \mathrm{mg} / \mathrm{day})$. To exploit a synergistic effect, ${ }^{3}$ IVT colistin $(60,000$ IU twice daily for 2 days, then escalated to 120,000 IU twice daily) and IV meropenem ( $2 \mathrm{~g}$ thrice daily) were added. This therapy led to an improvement in the meningitis. After 2 weeks, however, there was a progressive worsening of neurological conditions without fever or chemical CSF changes. The patient became confused, complaining of bilateral visual loss and mild weakness in the lower extremities; then, drowsiness and signs of severe myelitis appeared (paraplegia, severe weakness of upper extremities, loss of sensation at the T-7 level, and urinary retention). Brain and spine MRI showed ventriculitis and holocord myelitis (Fig. 2A and C). Immediately, IVT therapy with both the tigecycline and the colistin was stopped (45 days from the start of IVT tigecycline) while the IV tigecycline and meropenem were continued. Dexamethasone (12 mg/day) was added. In the following days, clinical signs of myelitis improved, but the meningitis recurred. Therefore, because of the known neurotoxicity of IVT colistin, ${ }^{6,9}$ we decided to restart the IVT tigecycline only, at a dose of $4 \mathrm{mg} /$ day. The signs of meningitis disappeared in a few days, and the Acinetobacter CSF load steadily decreased until CSF sterilization. Thus, after 1 month from the restart of the IVT tigecycline and after 1 week of persistently negative CSF cultures, the ventricular catheter was removed and the antibiotic therapy was discontinued. The patient was sent to a rehabilitation unit.

\section{Follow-Up}

At the 12-month follow-up, the patient was in good clinical condition without signs of CSF infection. Neurologically, he was alert and perfectly oriented; visual acuity 
TABLE 1. Antimicrobial susceptibility testing for Acinetobacter calcoaceticus-baumannii complex in CSF

\begin{tabular}{lrc}
\hline \multicolumn{1}{c}{ Antimicrobial Agent } & MIC $(\mu \mathrm{g} / \mathrm{ml})$ & Susceptibility* \\
\hline Tigecycline & 2 & NA \\
\hline Amoxicillin-clavulanic acid & $\geq 32$ & Resistant \\
\hline Piperacillin-tazobactam & $\geq 128$ & Resistant \\
\hline Cefotaxime & $\geq 64$ & Resistant \\
\hline Ceftazidime & $\geq 64$ & Resistant \\
\hline Cefepime & $\geq 64$ & Resistant \\
\hline Ertapenem & $\geq 8$ & Resistant \\
\hline Imipenem & $\geq 16$ & Resistant \\
\hline Gentamicin & $\geq 16$ & Resistant \\
\hline Ciprofloxacin & $\geq 4$ & Resistant \\
\hline Ampicillin & $\geq 32$ & Resistant \\
\hline Trimethoprim-sulfamethoxazole & $\geq 320$ & Resistant \\
\hline Colistin & 32 & Resistant \\
\hline
\end{tabular}

$\mathrm{MIC}=$ minimum inhibitory concentration; NA = not applicable.

* Susceptibility was defined according to the EUCAST criteria.

in the right eye was 20/20 with no visual field deficits; in the left eye, hand motion was visible at $20 \mathrm{~cm}$; strength in the upper limbs was normal; the patient was able to walk with a cane; sensory examination was unremarkable; and intermittent urinary catheterization was still necessary. Radiological signs of neuroinflammation had disappeared (Fig. 2B and D).

\section{Discussion}

In the present paper, we report on the successful treatment of severe postoperative meningitis from Acinetobacter baumannii using intraventricularly injected tigecycline-such a therapeutic use had not been recommended in the medical literature. We think that our experience, suggesting practical hints regarding route, frequency, and dosage of administration, could be of help in managing these tremendous infections.

The current treatment of MDR or XDR Acinetobacter meningitis relies mainly on colistin, a polymyxin-derived antibiotic. ${ }^{8}$ Polymyxins have been available since the 1950 s, but their use decreased in the 1980s because of serious nephro- and neurotoxicity., ${ }^{4,5,7,8}$ Currently, there is renewed interest in these drugs because they have retained activity against MDR and XDR gram-negative bacteria, including Acinetobacter., ${ }^{4,5}$, Since colistin has limited CNS penetration, the IVT route has been attempted in recent years. ${ }^{1,2,5,8}$ The IVT administration of antibiotics is largely used with favorable outcomes ${ }^{13}$ despite the lack of prospective randomized studies. In this context, the European Commission has recently extended the authorization for colistin use in serious infections from resistant bacteria and has included recommendations for IVT administration. ${ }^{4}$ Nonetheless, neurotoxicity from IVT colistin is a well-known occurrence, with a reported rate up to $21.7 \%$. $^{6}$ Chemical ventriculitis, chemical meningitis, and seizures are the most frequent adverse events. No deaths directly linked to IVT colistin administration have been reported, although 1 case of cauda equina syndrome has been de-
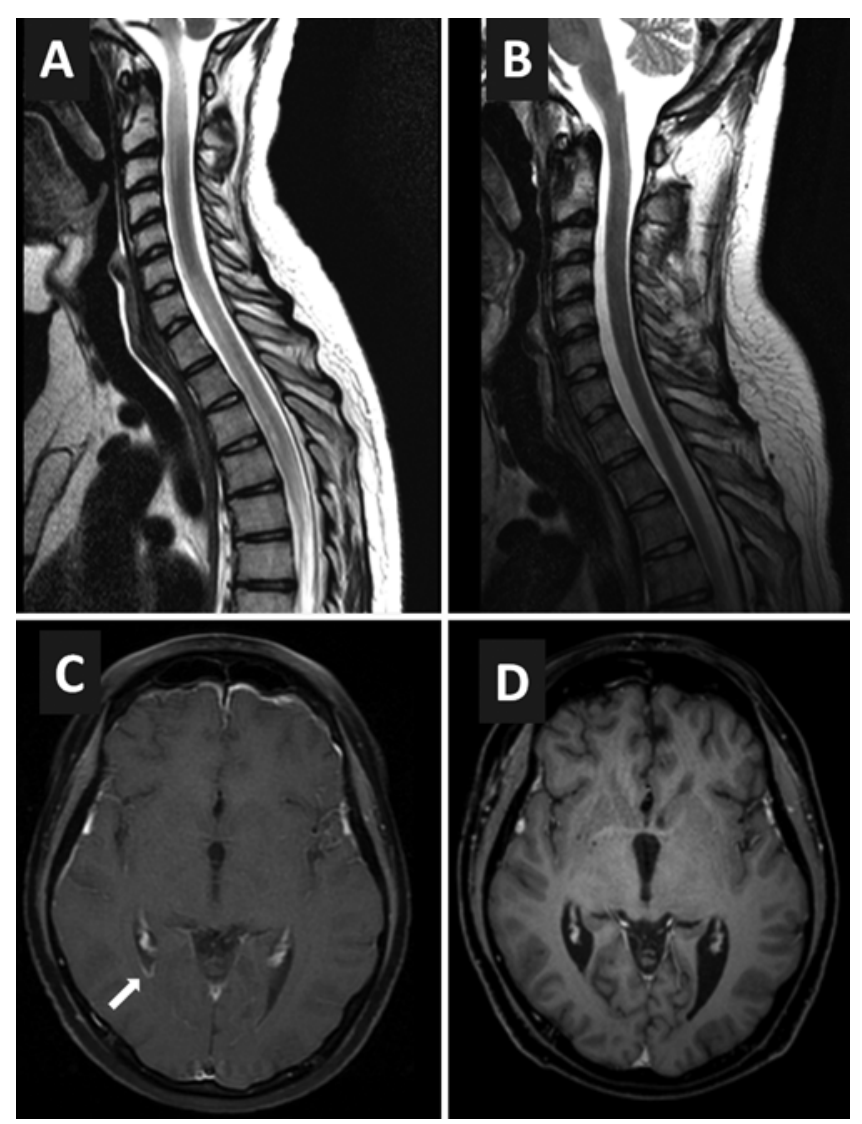

FIG. 2. Inflammatory MRI changes due to neurotoxicity. Spinal cord and brain MR images obtained when neurological conditions deteriorated (3 weeks after IVT colistin had been added), showing diffuse hyperintensity of the spinal cord on a sagittal T2 image as a sign of myelitis $(\mathbf{A})$ and leptomeningeal and ventricular (particularly trigonal, arrow) contrast enhancement (C). Spinal cord and brain MR images obtained 3 months after discharge, showing resolution of the myelitis with disappearance of cord T2 hyperintensity (B) and disappearance of the leptomeningeal and ventricular enhancement (D).

scribed. ${ }^{9}$ Moreover, the onset of colistin-resistant Acinetobacter strains is a well-recognized and potentially hazardous event, although such reports have been sporadic.?

Tigecycline is the first clinically available compound of glycylcyclines, a novel class of antibiotics designed to overcome drug resistance. ${ }^{12}$ The clinical use of IV tigecycline was approved in the US in 2005 and in the European Union in 2006. Reportedly, tigecycline has activity against vancomycin-resistant enterococci, methicillin-resistant Staphylococcus aureus, penicillin-resistant Streptococcus pneumoniae, and many species of MDR gram-negative bacteria. ${ }^{12}$ Thus, tigecycline has an excellent activity against Acinetobacter strains, in both the preclinical ${ }^{12}$ and clinical setting. ${ }^{5}$ Moreover, it has shown synergistic activity with a number of antimicrobial agents, including colistin. ${ }^{3}$ Tigecycline has a favorable toxicity profile, particularly regarding nervous system adverse events. ${ }^{12}$ As a counterpoint, its penetration into the CNS is quite low (around 11\%,11,14); therefore, it is not currently recommended for Acinetobacter meningitis. ${ }^{8}$ In summary, tigecycline, because of its excellent effectiveness against XDR bacteria, its safety profile, and limited CNS penetration, 
is an ideal candidate for IVT use. In a literature review, we found a single study in which the intrathecal administration of tigecycline was allegedly used to treat a case of postsurgical meningitis from Elizabethkingia meningoseptica ${ }^{15}$ however, details on the administration schedule as well as the treatment outcome were not available.

In our patient, tigecycline was administered intraventricularly because of a life-threatening infection and the absence of alternative therapies after intravenous therapy alone had failed. As with other IVT antibiotics, ${ }^{10}$ the initial IVT tigecycline dose of $2 \mathrm{mg} /$ day was calculated as $1 / 100$ of a daily IV dose ( $200 \mathrm{mg} /$ day $)$; later, the dose could be safely escalated to $4 \mathrm{mg} / \mathrm{day}$. This treatment took a long time to be effective-more than 2 months overall. In the meantime, an attempt to add IVT colistin resulted in neurotoxicity with severe myelotoxicity. Colistin neurotoxicity has been reported. ${ }^{6}$ The role of IVT tigecycline in such an adverse event can be reasonably excluded in our case because of the timeline of neurological deficits, which appeared only after the start of the IVT colistin, gradually improved once the colistin was stopped, and continued to improve after the tigecycline was restarted. Noticeably, at the 12-month follow-up, neurological conditions had markedly improved and our patient had started to walk again with assistance.

The use of IVT tigecycline, if confirmed to be safe and effective in other reports, could be considered as first-line therapy in patients with CNS infections from XDR bacteria resistant to colistin and susceptible to tigecycline (mainly, but not limited to, Acinetobacter spp. or Klebsiella spp.) or as second-line therapy in patients with infections from susceptible bacteria who have reported adverse reactions to the intrathecal administration of colistin.

\section{Conclusions}

In summary, IVT tigecycline should be regarded as a valuable tool against MDR CNS infections.

\section{References}

1. De Bonis P, Lofrese G, Scoppettuolo G, Spanu T, Cultrera R, Labonia M, et al: Intraventricular versus intravenous colistin for the treatment of extensively drug resistant Acinetobacter baumannii meningitis. Eur J Neurol 23:68-75, 2016

2. De Pascale G, Pompucci A, Maviglia R, Spanu T, Bello G, Mangiola A, et al: Successful treatment of multidrug-resistant Acinetobacter baumannii ventriculitis with intrathecal and intravenous colistin. Minerva Anestesiol 76:957-960, 2010

3. Entenza JM, Moreillon P: Tigecycline in combination with other antimicrobials: a review of in vitro, animal and case report studies. Int J Antimicrob Agents 34:8.e1-8.e9, 2009

4. European Commission: Commission Implementing Decision of 16.12.2014 concerning, in the framework of Article 31 of Directive 2001/83/EC of the European Parliament and of the Council, the marketing authorisations of medicinal products for human use which contain the active substance "colistimethate sodium and colistin" (16.12.2014 C(2014) 10081 final). Brussels: European Commission, 2014
5. Karageorgopoulos DE, Falagas ME: Current control and treatment of multidrug-resistant Acinetobacter baumannii infections. Lancet Infect Dis 8:751-762, 2008

6. Karaiskos I, Galani L, Baziaka F, Giamarellou H: Intraventricular and intrathecal colistin as the last therapeutic resort for the treatment of multidrug-resistant and extensively drug-resistant Acinetobacter baumannii ventriculitis and meningitis: a literature review. Int J Antimicrob Agents 41:499-508, 2013

7. Kempf M, Rolain JM: Emergence of resistance to carbapenems in Acinetobacter baumannii in Europe: clinical impact and therapeutic options. Int J Antimicrob Agents 39:105114,2012

8. Kim BN, Peleg AY, Lodise TP, Lipman J, Li J, Nation R, et al: Management of meningitis due to antibiotic-resistant Acinetobacter species. Lancet Infect Dis 9:245-255, 2009

9. Kim K, Kang HS, Sohn CH, Oh BM: Cauda equina syndrome misdiagnosed as aggravated hydrocephalus: neurological complication of intrathecal colistin in post-surgical meningitis. Acta Neurochir (Wien) 153:425-427, 2011

10. Nau R, Sörgel F, Eiffert H: Penetration of drugs through the blood-cerebrospinal fluid/blood-brain barrier for treatment of central nervous system infections. Clin Microbiol Rev 23:858-883, 2010

11. Pallotto C, Fiorio M, D'Avolio A, Sgrelli A, Baldelli F, Di Perri G, et al: Cerebrospinal fluid penetration of tigecycline. Scand J Infect Dis 46:69-72, 2014

12. Pankey GA: Tigecycline. J Antimicrob Chemother 56:470-480, 2005

13. Remeš F, Tomáš R, Jindrák V, Vaniš V, Setlík M: Intraventricular and lumbar intrathecal administration of antibiotics in postneurosurgical patients with meningitis and/or ventriculitis in a serious clinical state. J Neurosurg 119:1596-1602, 2013

14. Rodvold KA, Gotfried MH, Cwik M, Korth-Bradley JM, Dukart G, Ellis-Grosse EJ: Serum, tissue and body fluid concentrations of tigecycline after a single $100 \mathrm{mg}$ dose. J Antimicrob Chemother 58:1221-1229, 2006

15. Tak V, Mathur P, Varghese P, Misra MC: Elizabethkingia meningoseptica: an emerging pathogen causing meningitis in a hospitalized adult trauma patient. Indian J Med Microbiol 31:293-295, 2013

\section{Disclosures}

The authors report no conflict of interest concerning the materials or methods used in this study or the findings specified in this paper.

\section{Author Contributions}

Conception and design: D'Alessandris, Lauretti, Fantoni, Pallini, Scoppettuolo. Acquisition of data: all authors. Analysis and interpretation of data: all authors. Drafting the article: all authors. Critically revising the article: Lauretti, Pallini, Scoppettuolo. Reviewed submitted version of manuscript: all authors. Approved the final version of the manuscript on behalf of all authors: D’Alessandris.

\section{Correspondence}

Quintino Giorgio D'Alessandris, Institute of Neurosurgery, Catholic University School of Medicine, Largo A. Gemelli, 8, Rome 00168, Italy.email: giorgiodal@ hotmail.it. 